\title{
EDITORIAL
}

\section{Diagnosis of culture negative endocarditis: novel strategies to prove the suspect guilty}

\author{
C K Naber, R Erbel
}

Heart 2003;89:241-243

Negative blood cultures can occur in up to a third of all cases of infective endocarditis, which often delays diagnosis and onset of treatment with profound impact on the clinical outcome. Thus novel strategies for the identification of culture negative cases are highly desirable

See end of article for authors' affiliations .....................

Correspondence to: Dr Christoph K Naber, Klinik für Kardiologie, Universitätsklinikum Essen, Hufelandstrasser 55 , 45122 Essen, Germany; Christoph.naber@ medizin.uni-essen.de
$\mathrm{T}$ he diagnosis of infective endocarditis with its multiple clinical and morphological manifestations remains a challenging task. The von Reyn criteria, published in 1981, focused mainly on clinical and pathological findings in combination with positive blood culture to diagnose infective endocarditis. ${ }^{1}$ They were helpful to standardise diagnostic criteria, but their positive and negative predictive values remained unacceptably low, especially in the absence of positive blood culture results. With the introduction of transoesophageal echocardiography for the diagnosis of infective endocarditis, ${ }^{2}$ and the implementation of this method into the diagnostic criteria by Durack and colleagues, ${ }^{3}$ sensitivity and specificity of the diagnosis was significantly increased. ${ }^{4}$ Yet, in culture negative cases, sensitivity of these Duke criteria remains limited. ${ }^{56}$

In most cases, there are two reasons for negative blood cultures: (1) patients received antibiotics before blood cultures are taken due to systemic infection or suspected diagnosis of a bacterial infection; and (2) the causative microorganisms have no, or limited proliferation in conventional blood cultures, or the diagnosis of the causative microorganisms requires special media or cell culture conditions. Negative blood cultures occur in 2.5-31\% of all cases of infective endocarditis, which often delays diagnosis and onset of treatment with profound impact on the clinical outcome. ${ }^{7}$ The difficulties arising from culture negativity in cases of suspected endocarditis may be illustrated by a recent example from our institution.

In November 2001, a 68 year old man was submitted to our department after syncope of unclear origin. The patient had undergone aortic valve replacement (Saint Jude 29.0 mm) in April 1999. Transthoracic and subsequent transoesophageal echocardiography yielded an oscillating structure of $3.8 \mathrm{~mm}$ length and $3.0 \mathrm{~mm}$ width at the aortic valve (fig 1). There was no concomitant valvar insufficiency and left ventricular (LV) function was normal. The patient had no clinical signs or symptoms of inflammation and routine blood cultures were negative. Since the anticoagulation management in this patient appeared inadequate, we diagnosed a thrombus at the aortic valve, and started intravenous anticoagulation with heparin and additional acetylsalicylic acid. After three weeks the control echocardiography showed no residual structure at the aortic valve, so the patient was switched to oral anticoagulation treatment and discharged.

Six months later, the patient was readmitted to our intensive care unit with severe dyspnoea (New York Heart Association functional class IV) and a new diastolic murmur. Echocardiography showed aortic insufficiency grade III caused by paravalvar leakage, a dilated LV with a reduced systolic function, and a relative mitral insufficiency grade II. This time the patient displayed moderate leucocytosis and $\mathrm{C}$ reactive protein elevation. Immediately, empiric antibiotic treatment was initiated, and the patient was submitted to urgent aortic valve re-replacement. Intraoperatively a perivalvar abscess was found at the aortic valve, and aortic root reconstruction was performed with pericardial patching and implantation of a Carpentier Edwards Perimount 27 mm prothesis. Under empiric antibiotic treatment, blood culture results and cultures of the aortic valve remained negative. However, inflammatory parameters returned to normal and the patient recovered quickly and was discharged after six weeks of antibiotic treatment.

Retrospectively, we suggest that the initially observed "thrombus" at the aortic valve represented already an endocarditic vegetation at the mechanical valve. In the following months, the inflammatory process then spread into the perivalvar tissue, with abscess formation and subsequent severe perivalvar insufficiency.

The above case illustrates that echocardiography can provide crucial diagnostic information. In fact, transoesophageal echocardiography appears essential for the diagnosis of culture negative endocarditis when the Duke criteria are applied. ${ }^{7}$

\section{MODIFYING THE DUKE CRITERIA}

However, in our patient application of the Duke criteria was not helpful. Only one major (echocardiography) and one minor criterion (predisposition) were found to be positive in this case. Following the original Duke criteria, these findings, together with a likely alternate diagnosis (thrombus), justifies the rejection of the diagnosis of infective endocarditis. Thus, modification of the Duke criteria appears highly desirable to improve sensitivity, especially in culture negative cases. One of the first modifications involved the clinical criteria proposed by Lamas and Eykyn. ${ }^{8}$ They were followed by the modifications proposed by Li and 


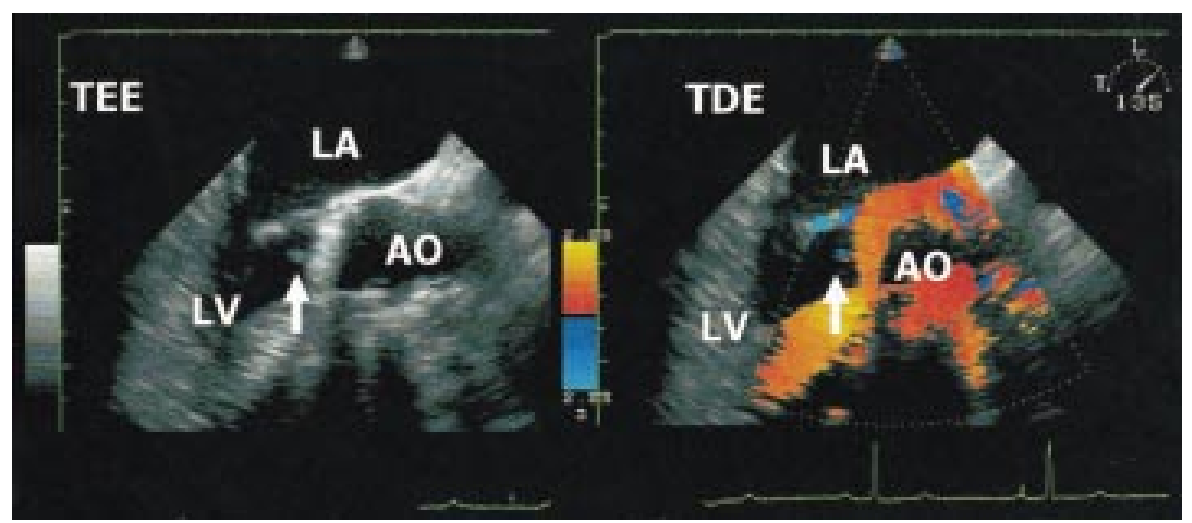

Figure 1 Oscillating structure of 3.8 $\mathrm{mm}$ length (arrow) at the aortic valve (Saint Jude $29 \mathrm{~mm}$ ) in a 68 year old patient with no signs and symptoms of inflammation and negative blood culture results. Left: transoesophageal echocardiography. Right: tissue Doppler echocardiography. A, aorta; $L A$, left atrium; $L V$, left ventricle.

Table 1 Duke criteria and modifications

\begin{tabular}{|c|c|c|}
\hline & Duke criteria & Suggested modifications \\
\hline Pathologic criteria & $\begin{array}{l}\text { 1. Microorganisms demonstrated by culture or histologic examination } \\
\text { 2. Active endocarditis demonstrated by histologic examination }\end{array}$ & \\
\hline Major criteria & $\begin{array}{l}\text { Positive blood cultures } \\
\text {-typical microorganisms consistent with endocarditis from two separate } \\
\text { blood cultures } \\
\text {-microorganisms consistent with endocarditis from persistently positive } \\
\text { blood cultures } \\
\text { Evidence of endocardial involvement } \\
\text {-echocardiography: oscillating structures, abscess formation, new partial } \\
\text { dehiscence of prosthetic valve } \\
\text {-new valvar regurgitation }\end{array}$ & 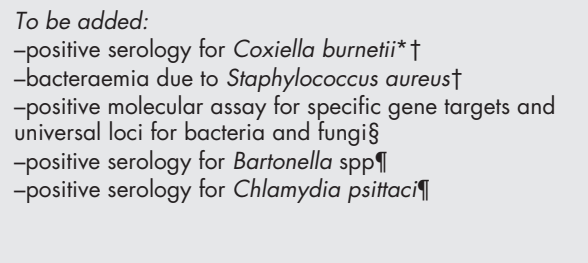 \\
\hline Minor criteria & $\begin{array}{l}\text {-predisposing heart disease } \\
\text {-fever }>38^{\circ} \mathrm{C} \\
\text {-vascular phenomena } \\
\text {-immunological phenomena } \\
\text {-microbiological evidence (no major criterion) } \\
\text {-suspect echocardiography (no major criterion) }\end{array}$ & $\begin{array}{l}\text { To be omitted: } \\
\text { Suspect echocardiography (no major criterion)* } \\
\text { To be added: } \\
\text { Elevated CRP, elevated ESR, splenomegaly, haematuria, } \\
\text { clubbing, splinter haemorrhagia, petechiae and purpurał }\end{array}$ \\
\hline
\end{tabular}

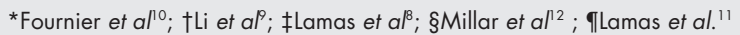

CRP, C reactive protein; ESR, erythrocyte sedimentation rate.

colleagues, ${ }^{9}$ considering, for example, a positive serology for Coxiella burnetti as a major criterion, which had already been proposed earlier by Fournier and colleagues ${ }^{10}$ (tables 1 and 2). Although these modifications were not specifically aimed at culture negative endocarditis, the changes proposed by Li and colleagues ${ }^{9}$ would at least have led to the diagnosis of possible endocarditis in our patient.

In this issue of Heart, Lamas and Eykyn ${ }^{11}$ present a study which investigates the value of the modified Duke criteria ${ }^{9}$ and their own St Thomas modifications of the Duke criteria, ${ }^{8}$ focusing specifically on culture negative endocarditis. They show that in the case of pathological proven native valve endocarditis with negative blood cultures, only $21 \%$ were clas- sified as definite by the original Duke criteria, 32\% were definite by the modified Duke criteria, while the St Thomas modifications correctly classified $62 \%$ as definite. A very interesting point in this observation is that the four cases that were additionally classified correctly by the modified Duke criteria were upgraded by considering a positive serology for $C$ burnetti as a major criterion. In addition, the authors demonstrate that a "closer look" may lead to the identification of the causative organism in a notable number $(49 \%)$ of culture negative cases. This was achieved by serology (C burnetti, Bartonella species, Chlamydia psittaci) in $24 \%$, by culture of the excised valve in $14 \%$, by microscopy of the excised valve in $5 \%$, and by culture from a site other than the excised valve in $6 \%$.

Table 2 Original and modified diagnostic categories by the Duke criteria

\begin{tabular}{lll}
\hline Categories & Original Duke criteria & Suggested modifications \\
\hline Definite & $\begin{array}{l}\text { Pathological criteria positive } \\
\text { or } 2 \text { major criteria positive } \\
\text { or } 1 \text { major and } 2 \text { minor criteria positive } \\
\text { or } 5 \text { minor criteria positive }\end{array}$ & 1 major and 1 minor criterion positive* \\
Possible & All cases which can not be classified as definite or rejected & 3 minor criteria positive* \\
Rejected & Alternate diagnosis & \\
& Resolution of the infection with antibiotic treatment for $\leqslant 4$ days & \\
\hline *Li et al. ${ }^{9}$ & No histological evidence &
\end{tabular}


In 2001, a similar "close look" approach had been emphasised by Millar and colleagues. ${ }^{12}$ They proposed to add a molecular approach of polymerase chain reaction amplification of specific gene targets and universal loci for bacteria and fungi and subsequent sequencing to identify the possible causative microorganisms in blood culture and heart valve material, as a major criterion to the Duke criteria. This proposal is nicely adapted by the work of Grijalva and colleagues also presented in this issue of Heart. ${ }^{13}$ They present a clinical validation of molecular methods for the diagnosis of infective endocarditis in culture negative cases undergoing surgery. They investigated valvar specimens of 15 patients with definite, but culture negative, endocarditis and of 13 controls without endocarditis. They show that in $93 \%$ of the culture negative cases the causative pathogen could be detected by using a molecular approach, while all controls remained negative.

\section{IDENTIFYING THE CAUSATIVE PATHOGEN}

It is evident that the identification of the causative pathogen, either by serology, microscopy, and additional culture, as proposed by Lamas an Eykyn, ${ }^{11}$ or by a molecular approach as demonstrated by Grijalva and colleagues, ${ }^{13}$ will improve the specificity of the therapeutic regimen and may significantly improve patient outcome. Specificity of the antibiotic treatment may be even more improved by including the investigation of common antimicrobial resistance genes into the molecular approach. ${ }^{14}$ This would allow the antibiotic treatment to be adapted according to the expected bacterial resistance.

In conclusion, modification of the original Duke criteria is necessary to enhance the diagnostic sensitivity, especially in culture negative cases; besides clinical signs and symptoms, ${ }^{8}$ serologic tests have been already added. ${ }^{90}$ These tests should be expanded to microorganisms other than $C$ burnetti, and microscopy and additional microbial cultures should be performed. Moreover, molecular analysis appears crucial in culture negative cases, and has been recently implemented into the newest revision of the Duke criteria. ${ }^{15}$ These additional features may not only improve the sensitivity of the diagnosis, but may also improve the general outcome of the patients by increasing the specificity of the antibiotic treatment.

Naturally, the number of cases investigated in all studies assessing the value of the Duke criteria and its respective modifications is, to date, relatively low. Even the largest studies include only around 100 patients. ${ }^{4}$ For the validation of the numerous factors that have been proposed to improve the sensitivity of the original criteria, larger populations will be necessary to increase the statistical power of the analyses. Such large populations, however, preclude a single centre's experience. The initiation of a large scale (for example, European) registry for cases of suspected endocarditis is, therefore, highly desirable.

\section{Authors' affiliations}

C K Naber, R Erbel, Klinik für Kardiologie, Universitätsklinikum Essen, Germany

\section{REFERENCES}

1 von Reyn FC, Arbeit RD, Friedland GH, et al. Criteria for the diagnosis of infective endocarditis. Clin Infect Dis 1994;19:368-70.

2 Erbel R, Rohmann S, Drexler $M$, et al. Improved diagnostic value of echocardiography in patients with infective endocarditis by transoesophageal approach. A prospective study. Eur Heart J 1988;9:43-53

3 Durack DT, Lukes AS, Bright DK. New criteria for diagnosis of infective endocarditis: utilization of specific echocardiographic findings. Duke endocarditis service. Am J Med 1994:96:200-9.

4 Naber CK, Bartel T, Eggebrecht H, et al. Diagnosis of endocarditis today: Duke criteria or clinical judgment? Herz 2001;26:379-90.

5 Kupferwasser LI, Bayer AS. Culture-negative endocarditis: etiology, diagnosis, management and therapy. Herz 2001;26:398-408.

6 Habib G, Derumeaux G, Avierinos JF, et al. Value and limitations of the Duke criteria for the diagnosis of infective endocarditis. J Am Coll Cardiol 1999;33:2023-9

7 Kupferwasser I, Darius H, Müller A, et al. Diagnosis of culture negative endocarditis: the role of the Duke criteria and the impact of transesophageal echocardiography. Am Heart J 2001;142:146-52.

8 Lamas CC, Eykyn SJ. Suggested modifications to the Duke criteria for the clinical diagnosis of native valve and prosthetic valve endocarditis: analysis of 118 pathologically proven cases. Clin Infect Dis 1997:25:713-19.

9 Li JS, Sexton DJ, Mick N, et al. Proposed modifications to the Duke criteria for the diagnosis of infective endocarditis. Clin Infect Dis 2000;30:633-8.

10 Fournier PE, Casalta JP, Habib G, et al. Modification of the diagnostic criteria proposed by the Duke Endocarditis Service to permit improved diagnosis of Q fever endocarditis. Am J Med 1996;100:629-33.

11 Lamas CC, Eykyn SJ. Blood culture negative endocarditis: analysis of 63 cases presenting over 25 years. Heart 2003;89:258-62.

12 Millar BC, Moore JE, Mallon P, et al. Molecular diagnosis of infective endocarditis - a new Duke's criterion. Scand J Infect Dis 2001;33:673-80.

13 Grijalva $\mathbf{M}$, Horvath $R$, Dendis $M$, et al. Molecular diagnosis of culture negative infective endocarditis: clinical validation in a group of surgically treated patients. Heart 2003:89:263-8.

14 Moore JE, Millar BC, Yongmin X, et al. A rapid molecular assay for the detection of antibiotic resistance determinants in cause of infective endocarditis. J Appl Microbiol 2001;90:719-26.

15 Lisby G, Gutschik E, Durack DT. Molecular methods for diagnosis of infective endocarditis. Infect Dis Clin North Am 2002;16:393-412. 